\title{
A Study Of Efficacy And Safety With Apatinib Or Apatinib Combined With Chemotherapy In Recurrent/advanced Ovarian Cancer Patients
}

This article was published in the following Dove Press journal: Cancer Management and Research

\author{
Mi Yang ${ }^{1,2}$ \\ Xiufeng Liu $\mathbb{D D}^{3}$ \\ Cheng Zhang ${ }^{4}$ \\ Feng Liao ${ }^{3}$ \\ Zixiong $\mathrm{Li}^{3}$ \\ Xianwen Luo ${ }^{3}$ \\ Yiran Sun ${ }^{5}$ \\ Chao Chen ${ }^{3}$
}

'The Comprehensive Cancer Center of Drum Tower Hospital, Medical School of Nanjing University, Nanjing 210008,

People's Republic of China; ${ }^{2}$ Cancer Center of Nanjing Gaochun People's Hospital, Nanjing 2I I300, People's Republic of China; ${ }^{3}$ Department of Medical Oncology of PLA Cancer Center, Jinling Hospital, Medical School of Nanjing University, Nanjing 210002, People's Republic of China; ${ }^{4}$ Department of Gynecology, Jiangsu Province Hospital of Chinese Medicine, Nanjing 210029 People's Republic of China; ${ }^{5}$ Department of Clinical Medicine, Xuzhou Medicine University, Xuzhou 221004, People's Republic of China
Correspondence: Chao Chen; Xiufeng Liu Department of Medical Oncology of PLA Cancer Center, Jinling Hospital, Medical School of Nanjing University, Nanjing 210002, People's Republic of China

Tel +86258086769

Email njjloncologycc@।63.com;

njjloncologylxf@163.com
Objectives: Despite recent advances in the treatment of advanced ovarian cancer, drug selection after second-line chemotherapy has not been well studied. In this study, we retrospectively evaluated the effect and safety of apatinib as monotherapy or in combination with chemotherapy for the treatment of advanced ovarian cancer after second-line treatment.

Methods: We reviewed the medical records of patients from April 2016 to October 2018 with advanced ovarian cancer who received apatinib after failed second-line chemotherapy. Overall survival (OS) and progression-free survival (PFS) were calculated by the KaplanMeier method. Response rate (RR) and disease control rate (DCR) were evaluated using radiologic reports according to RECIST 1.1 criteria. Treatment-related adverse events were evaluated based on NCI-CTC version 4.0.

Results: Study concerned 22 evaluated cases; of them, 13 patients received apatinib combined with chemotherapy and 9 patients received apatinib monotherapy. The median PFS was 8.2 months (9.7 months in combined group and 4.4 months in monotherapy group, $\mathrm{P}$ value was 0.21 ). The median OS was 13.1 months (13.6 months in combined group and 11.6 months in monotherapy group, P value was 0.45 ). The RR was $20 \%$ and DCR was $85 \%$ (combined group: RR 33.3\%, DCR 100\%, monotherapy group: RR 0\%, DCR 62.5\%). The main side effect was hypertension $(9 / 22)$, proteinuria $(7 / 22)$, oral mucositis $(5 / 22)$, hand and foot syndrome $(6 / 22 \%)$, leukopenia $(5 / 22)$, etc.

Conclusion: Apatinib showed good efficacy and safety for advanced ovarian cancer patients whether used alone or in combination with chemotherapy. In the meanwhile, this study is limited by the small cases number. Therefore, further research is needed to provide more data and ultimately apply it to guide clinical practice.

Keywords: apatinib, chemotherapy, ovarian cancer, efficacy and safety

\section{Introduction}

Ovarian cancer is the most common malignant tumor in gynecological tumors. In 2018, there were 295,414 new cases and 184,779 deaths worldwide. ${ }^{1}$ There were 22,240 new cases and 14,070 deaths in the United States; more than 95\% of ovarian cancer patients died at the age of over 45 years old. ${ }^{2}$

There were 52,100 new cases and 22,500 deaths in China in 2015. ${ }^{3}$ Surgical treatment combined with platinum chemotherapy is the preferred treatment, but about $80 \%$ of patients with ovarian cancer will have recurrence and metastasis after standard treatment. ${ }^{4}$ There was a lack of evidence-based medical guideline for drug selection for patients who failed after second-line chemotherapy. Apatinib is a small 
molecule vascular endothelial growth receptor inhibitor. It inhibits angiogenesis and exerts an anti-tumor effect mainly by highly selectively inhibiting the activity of vascular endothelial growth factor receptor 2 (VEGFR-2) tyrosine kinase and blocking the signal transduction pathway of vascular endothelial growth factor (VEGF) binding to its receptor. Apatinib is currently mainly used to treat gastric cancer and liver cancer. ${ }^{5-7}$ Antiangiogenic therapy has been shown to be an attractive therapeutic strategy for ovarian cancer; bevacizumab and Pazopanib statistically significantly improved PFS. ${ }^{8,9}$

Our team reviewed medical records of patients with advanced ovarian cancer who received apatinib after second-line treatment. According to this retrospective study, we compared the efficacy of apatinib monotherapy and apatinib combined with chemotherapy in the treatment of advanced ovarian cancer for the first time, and reported apatinib-related side effects.

\section{Materials And Methods General Data Collection}

All ovarian cancer patients who received apatinib after second-line chemotherapy in Jinling Hospital and Nanjing Drum Tower Hospital of Nanjing University between April 2016 and Oct 2018 were considered for inclusion in this retrospective study. We reviewed the database and medical records to extract clinicopathologic data including age, Eastern Cooperative Oncology Group performance status (ECOG), histologic type, prior therapy, symptoms, laboratory results, image reports, etc. We excluded patients whose apatinib treatment period was too short (less than 3 months of apatinib) or patients without follow-up.

This study was approved by the Ethics Committee of Jinling Hospital and Nanjing Drum Tower Hospital of Nanjing University. Owing to the retrospective study design and analysis of clinical data, informed consent was formally waived by the Ethics Committee of Jinling Hospital and Nanjing Drum Tower Hospital of Nanjing University. All patient information is ensured to be confidential. All the procedures in this study are in accordance with the Helsinki Declaration.

\section{Efficacy Assessment And Adverse Event Assessment}

CT imaging was used to evaluate tumor assessments by oncologists and imaging specialists. According to RECIST
1.1, the efficacy was divided into complete response (CR), partial response (PR), stable disease (SD) and progressive disease (PD). The overall response rate (RR) was calculated by $\mathrm{CR}+\mathrm{PR}$ and the disease control rate (DCR) was calculated by $\mathrm{CR}+\mathrm{PR}+\mathrm{SD}$. Adverse events were evaluated based on the National Cancer Institute Common Toxicity Criteria (NCI-CTC) version 4.0.

The overall survival (OS) was defined as the duration between the date of treatment initiation to the date of death or last follow-up, with patients alive at last follow-up censored on that date. We defined progression-free survival (PFS) from the date of the first recurrence to the date of second recurrence or death, with patients censored on the date of the last follow-up, if the patients were without recurrence on that date. Survival data were calculated by the Kaplan-Meier method.

\section{Statistical Methods}

All statistical data were analyzed by SPSS 20.0 professional statistical software; all the count data were expressed as a percentage (\%), and the survival curve was drawn by Kaplan-Meier method for prognosis follow-up.

\section{Results}

\section{General Data And Treatment}

Thirty patients were treated with apatinib (Figure 1). One patient had a survival of fewer than 3 months, and 4 refused to take apatinib for its toxicity. Three patients had no follow-up. A total of 22 patients were analyzed. The median age of these patients was 55.6 years (35-79 years) (Table 1$)$. The ECOG was 1-3.

Of 22 patients, 13 patients received apatinib combined with chemotherapy and 9 patients received apatinib monotherapy. Apatinib mesylate (Jiangsu Hengrui Pharmaceutical Co., Ltd.) was orally administered $500 \mathrm{mg} /$ day as initial dose. When there was an adverse reaction of grade 3 or above, the dose of apatinib is reduced until the disease progresses or an intolerable adverse reaction occurs.

All of 22 patients received apatinib after second-line chemotherapy. Most of the patients received more than three lines chemotherapy. All of these patients progress to stage IV when they received apatinb treatment.

In this study, all of 22 patients received platinum-containing chemotherapy as the first-line treatment. After first-line treatment, the choices of second-line and chemotherapy were different, including taxol, paclitaxel, topotecan, gemcitabine, 


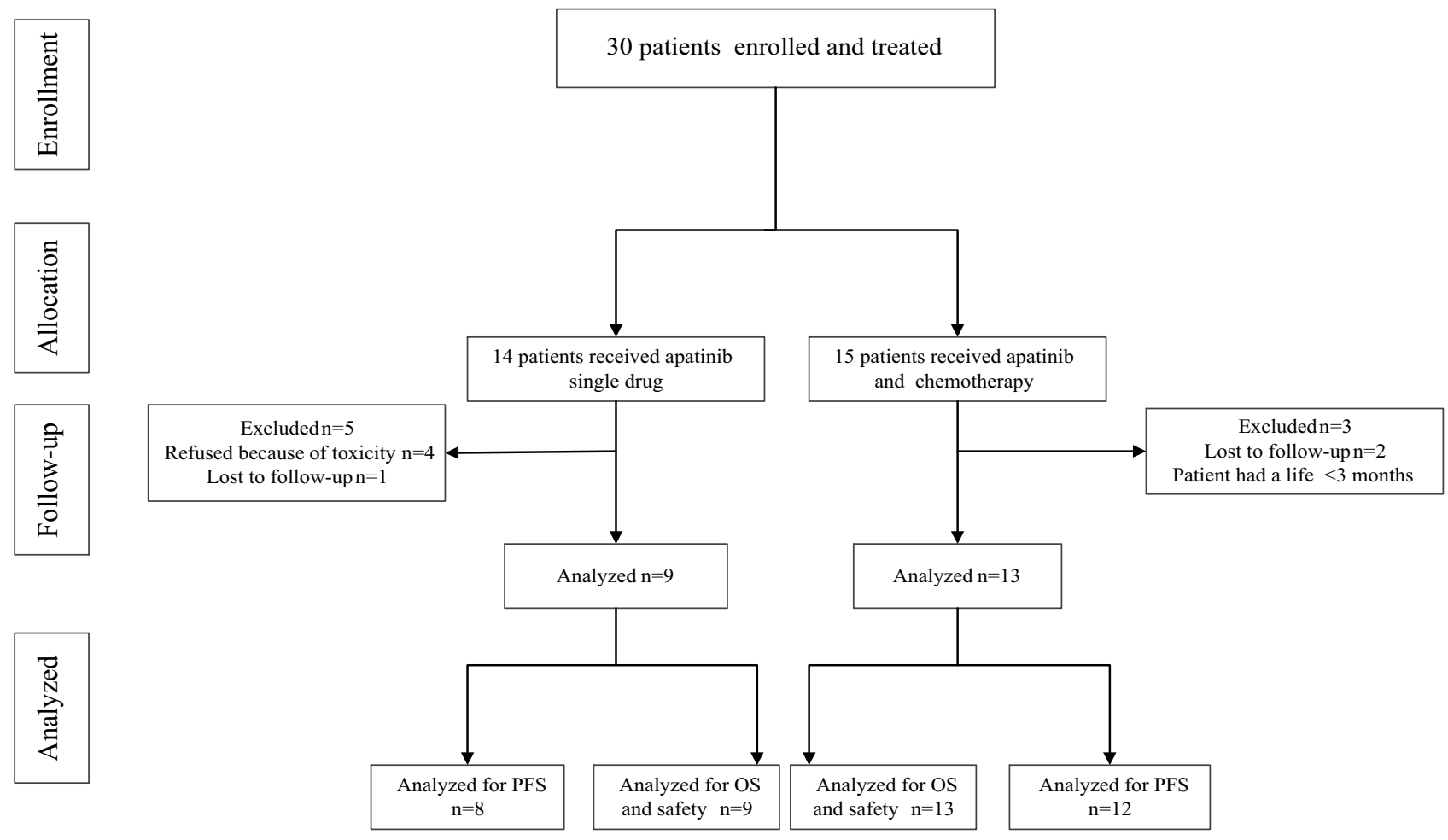

Figure I Trial profile.

5-fluorouracil, oxaliplatin, etc. So the combination chemotherapy drug was based on the patient's previous chemotherapy.

Table I Patient Demographics And Disease Characteristics

\begin{tabular}{|l|l|}
\hline Variable & No. Of Cases (\%) \\
\hline $\begin{array}{l}\text { Age, years } \\
\text { Median }\end{array}$ & 55.6 \\
Range & $35-79$ \\
\hline PS ECOG & \\
0 & $1(4.5 \%)$ \\
I & $16(72.7 \%)$ \\
2 & $4(18.2 \%)$ \\
3 & $1(4.5 \%)$ \\
\hline Histologic type & \\
Serous carcinoma & $14(63.6 \%)$ \\
Endometrioid carcinoma & $2(9.1 \%)$ \\
Clear cell carcinoma & $4(18.2 \%)$ \\
Not available & $2(9.1 \%)$ \\
\hline Lines of prior anticancer therapy & \\
I-3 & $6(27.3 \%)$ \\
$>3$ & $16(72.7 \%)$ \\
\hline Prior therapy & \\
Chemotherapy and surgery & $19(86.4 \%)$ \\
Surgery, radiotherapy and chemotherapy & $3(13.6 \%)$ \\
\hline
\end{tabular}

Note: All patients enrolled $(n=22)$.

\section{Clinical Efficacy}

Complete imaging data of 20 patients were obtained. Patients were evaluated by spiral CT every 6 weeks. Four cases for PR (4 cases in combined group, 0 in monotherapy group), 13 cases for SD (8 cases in combined group, 5 cases in monotherapy group), 3 cases for PD ( 0 cases in combined group, 3 patients in the monotherapy group), 4 patients were intolerant. RR 20\%, DCR 85\% (combined group: RR 33.3\%, DCR 100\%, monotherapy group: RR 0\%, DCR 62.5\%) (Table 2). Tumor shrinkage was noted in $8(8 / 20)$ patients (Figure 2).

The patient's overall mPFS was 8.2 months $(95 \% \mathrm{CI}$ : 4.93-11.42) (Figure 3A); mOS was 13.1 months (95\% CI: 10.1-16.0) (Figure 3B). The mPFS of combined group was 9.7 months (95\% CI: 5.42-14.06) and the mPFS of monotherapy group was 4.4 months (95\% CI: $2.48-6.38)$ with a $\mathrm{P}$ value of 0.21 (Figure 4A). The mOS of the combined group was 13.6 months (95\% CI: 10.52-16.71), and the mOS of monotherapy group was 11.6 months (95\% CI: 6.66-16.49) with a $\mathrm{P}$ value of 0.45 (Figure 4B).

\section{Adverse Reactions}

Safety analysis was performed on 22 patients. The main adverse reactions included 9 cases of hypertension ( 4 cases in the monotherapy group, 5 cases in the combined group), 
Table 2 Summary Of Tumor Response

\begin{tabular}{|l|l|l|l|}
\hline & Monotherapy, n (\%) & Combined Treatment, n (\%) & Total (\%) \\
\hline Complete response & 0 & 0 & 0 \\
Partial response & 0 & $4(33.3 \%)$ & $4(20 \% ; 8-39.2)$ \\
Stable disease & $5(62.5 \%)$ & $8(66.7 \%)$ & $13(60 \% ; 36.5-83.5)$ \\
Progressive disease & $3(37.5 \%)$ & 0 & $3(15 \% ;-2.1-32.1)$ \\
Response rate & $5(62.5 \%)$ & $12(100 \%)$ & $17(85 \% ; 67.9-100)$ \\
$($ CR+PR+SD) & & & \\
\hline
\end{tabular}

Notes: Data are $\mathrm{n}(\%)$ and $\mathrm{n}(\% ; 95 \% \mathrm{Cl})$. Two patients without post or baseline efficacy assessments were excluded.

1 case of heart failure (monotherapy group), 7 cases of proteinuria ( 1 case in the monotherapy group, 6 cases in the combined group), 5 cases of oral mucositis ( 2 cases in the monotherapy group, 3 cases in the combined group), 6 cases of skin reactions in the hands and feet ( 1 case in the monotherapy group, 5 cases in the combined group), 3 cases of bleeding ( 1 case in the monotherapy group, 2 cases in the combined group), 6 cases of fatigue ( 3 cases in the monotherapy group, 3 cases in the combined group), 5 cases of appetite loss ( 4 cases in the monotherapy group, 1 case in the combined group), 3 cases of edema ( 1 case in the monotherapy group, 2 cases in the combined group).

There were 5 cases of leukopenia ( 2 cases in the monotherapy group, 3 cases in the combined group), 2 cases of thrombocytopenia ( 1 case in the monotherapy group, 1 case in the combined group), 2 cases of anemia ( 1 case in the monotherapy group, 1 case in the combined group) (Table 3 ).

\section{Discussion}

First-line therapy of advanced ovarian cancer involves primary cytoreductive surgery and adjuvant systemic

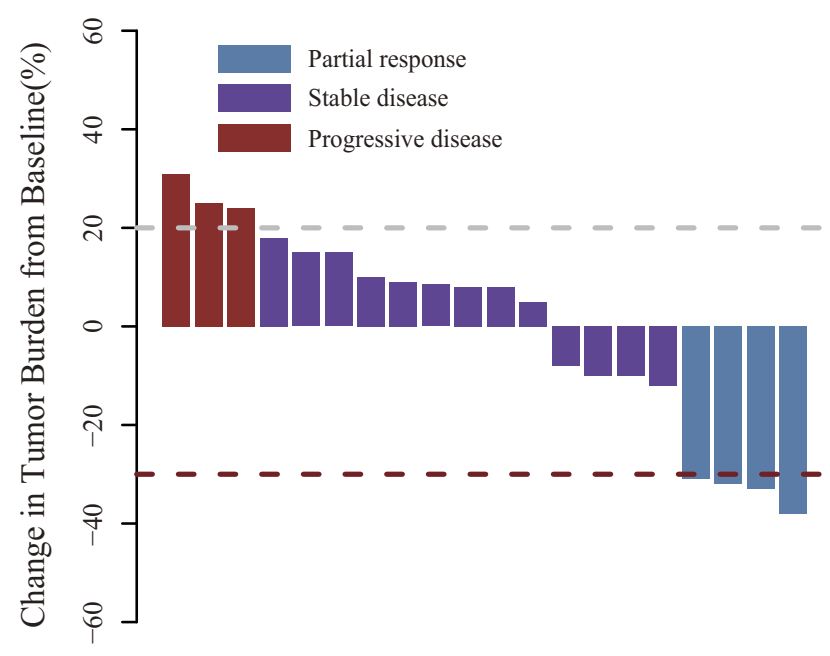

Figure 2 Waterfall plot for the best percentage change in target lesion size. chemotherapy (including platinum drugs). Progression of incompletely resected disease or recurrence after cytoreduction is inevitable. The approach to second-line or following treatment is ill-defined. The ovarian cancer
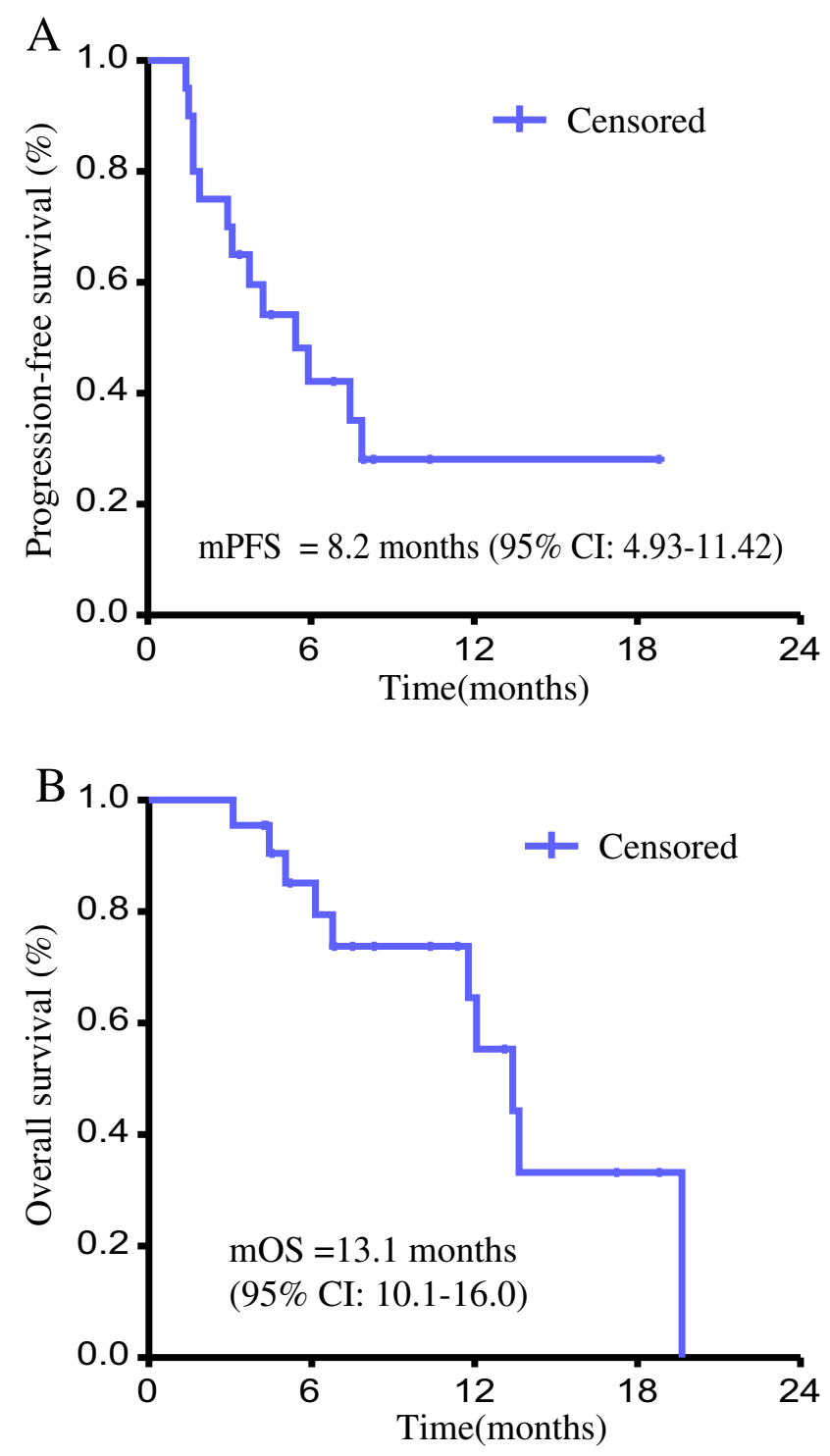

Figure 3 Progression-free survival (A) and overall survival (B) of patients with treated by apatinib. 

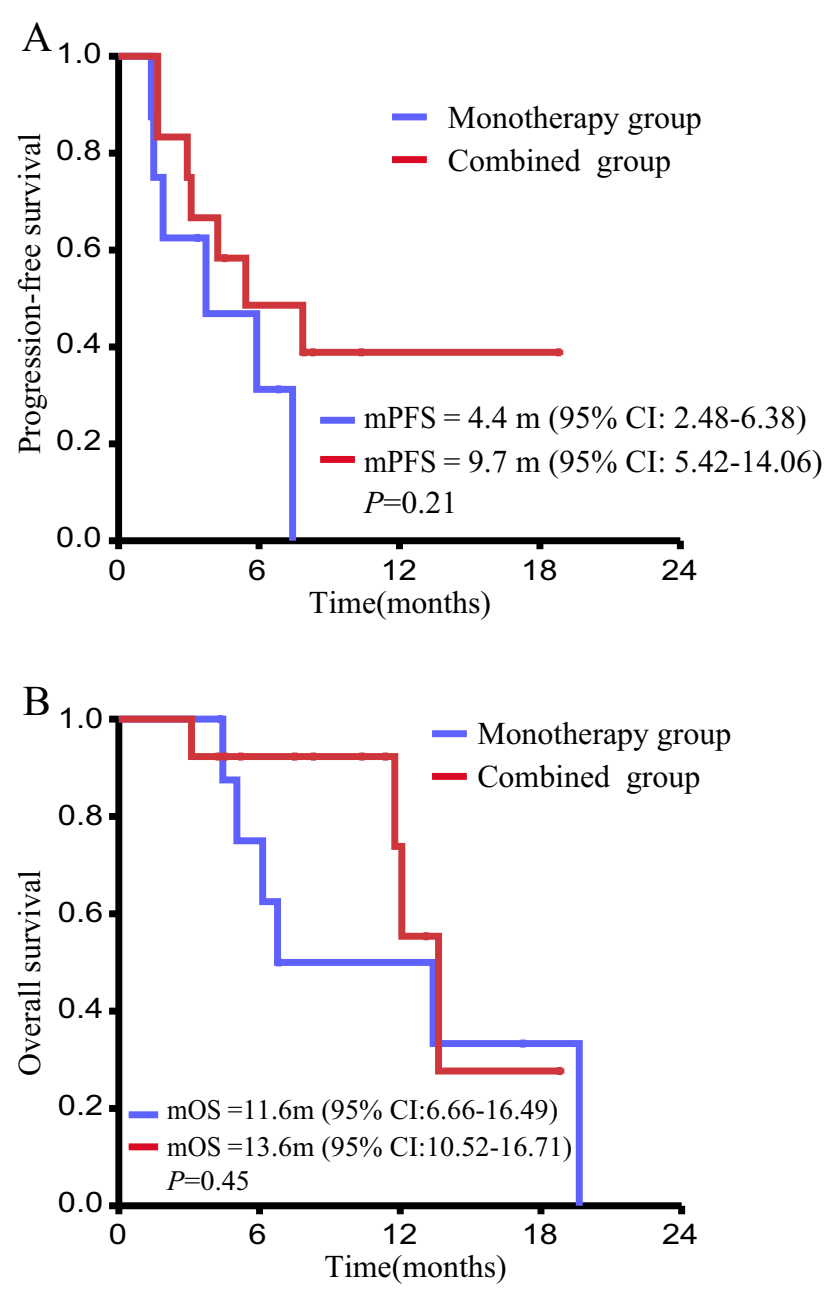

Figure 4 Progression-free survival (A) and overall survival (B) of patients received apatinib or apatinib combined with chemotherapy.

resistance to chemotherapy may be due to reduced immunosurveillance and drug-resistant cells. ${ }^{10,11}$

There are more and more applications for targeted therapy for ovarian cancer, including PARP inhibitors, $\mathrm{PI} 3 \mathrm{~K} / \mathrm{AKT} / \mathrm{mTOR}$ signaling pathway inhibitors and angiogenesis inhibitors. Tumors require more nutrients and oxygen in the process of abnormal proliferation, which requires transporting them from the host to the tumor tissue through the neovascularization, so angiogenesis inhibitors have become one of the critical research subjects. Since the VEGF/VEGFR-2 signaling pathway is an essential target for tumor anti-angiogenesis therapy, inhibition of tumor angiogenesis has become the focus of tumor-targeted treatment. ${ }^{12}$ Comparison of bevacizumabcombined chemotherapy (carboplatin + paclitaxel) with chemotherapy showed a significant increase in median PFS after maintenance therapy of bevacizumab (14.1 vs
10.3 months, $\mathrm{P}<0.001)$; there was no improvement in PFS in patients without bevacizumab maintenance therapy. ${ }^{13,14}$ OS in the bevacizumab-treated group was significantly improved (42.2 vs 37.3 months $\mathrm{P}=0.056$ ), and the incidence of adverse reactions in the combined group and chemotherapy group was 96\% (317/325) and 86\% (282/ 332), respectively. ${ }^{15}$

The SOLO2/ENGOTOv2 study showed that the PFS of the patients with advanced ovarian disease with BRCA1 mutation in the olaparib group and the placebo group after treatment were 19.1 months and 5.5 months, respectively ( $\mathrm{P}<0.0001) .{ }^{16}$ Rucaparib is another potent oral small molecule PARP inhibitor that is also most effective in patients with BRCA mutations. A total of 564 patients were enrolled in the ARIEL3 trial. PFS in patients with BRCA mutations in the rucaparib group was significantly longer than in the placebo group (16.6 months vs 5.4 months, $\mathrm{P}<0.0001)$. ${ }^{17}$ Niraparib is the third PARP inhibitor approved by the FDA following olaparib and rucaparib. The NOVA trial showed that niraparib was used as maintenance therapy for 553 patients with platinum-sensitive advanced ovarian cancer. The results showed that the PFS of the niraparib group in the germline BRCA (gBRCA) mutation cohort was significantly longer than that in the placebo group (21.0 months vs 5.5 months, $\mathrm{P}<0.001)$, and the PFS in the non-gBRCA cohort were 9.3 months and 3.9 months in both groups, respectively $(\mathrm{P}<0.001){ }^{17}$

Mirvetuximab Soravtansine (IMGN853) is a folate receptor alpha $(\mathrm{FR} \alpha)$-targeting antibody-drug conjugate (ADC). The efficacy and safety of platinum-resistant ovarian cancer have also been confirmed by clinical trials. ${ }^{18-20}$

Apatinib is an oral tyrosine kinase inhibitor of small molecule vascular endothelial growth factor receptor-2 (VEGFR-2) that competitively binds to the receptor's intracellular tyrosine ATP binding site, highly selectively inhibits VEGFR-2 tyrosine kinase activity, blocks downstream signal transduction and inhibits tumor angiogenesis, so as to treat tumors. ${ }^{5,21}$ In October 2014, it was approved by the China Food and Drug Administration (CFDA) for the third-line or above treatment of advanced gastric adenocarcinoma and gastric-esophageal junction adenocarcinoma. $^{22}$ The 2015 NCCN Clinical Practice Guide uses it as a second-line treatment for advanced gastric cancer. ${ }^{23}$ The clinical trials of apatinib for the treatment of various solid tumors such as breast cancer, lung cancer, advanced liver cancer and soft tissue sarcoma are underway at the same time. The comparative report of apatinib and apatinib combined with chemotherapy in the treatment of advanced ovarian cancer is rare. 
Table 3 Common Adverse Events And Clinical Laboratory Abnormalities

\begin{tabular}{|c|c|c|c|c|c|}
\hline \multirow[t]{2}{*}{ Adverse Event } & \multicolumn{2}{|c|}{ Grade I/2, n (\%) } & \multicolumn{2}{|c|}{ Grade $3 / 4, \mathrm{n}(\%)$} & \multirow[t]{2}{*}{ Total, n (\%) } \\
\hline & Monotherapy & Combined Treatment & Monotherapy & Combined Treatment & \\
\hline \multicolumn{6}{|l|}{ Non-hematologic } \\
\hline Hypertension & $2(9.1 \%)$ & $3(13.6 \%)$ & $2(9.1 \%)$ & $2(9.1 \%)$ & 9 (40.5\%) \\
\hline Mucositis & $2(9.1 \%)$ & $2(9.1 \%)$ & 0 & I (4.5\%) & 5 (22.7\%) \\
\hline Fatigue & $3(13.6 \%)$ & $3(13.6 \%)$ & 0 & 0 & $6(27.2 \%)$ \\
\hline Anorexia & $3(13.6 \%)$ & I (4.5\%) & I (4.5\%) & 0 & $5(22.7 \%)$ \\
\hline Albuminuria & I (4.5\%) & $5(22.7 \%)$ & 0 & I (4.5\%) & 7 (31.8\%) \\
\hline Hand foot syndrome & I (4.5\%) & $2(9.1 \%)$ & 0 & $3(13.6 \%)$ & $6(27.2 \%)$ \\
\hline Hemorrhage & I (4.5\%) & $2(9.1 \%)$ & 0 & 0 & $3(13.6 \%)$ \\
\hline Edema & I (4.5\%) & $2(9.1 \%)$ & 0 & 0 & $3(13.6 \%)$ \\
\hline Cardiac failure & 0 & 0 & I (4.5\%) & 0 & I (4.5\%) \\
\hline \multicolumn{6}{|l|}{ Hematologic } \\
\hline Hemoglobin & $2(9.1 \%)$ & 0 & 0 & 0 & $2(9.1 \%)$ \\
\hline Leukocyte & I (4.5\%) & $3(13.6 \%)$ & 0 & I (4.5\%) & 5 (22.7\%) \\
\hline Platelets & 0 & 0 & I (4.5\%) & I (4.5\%) & 2 (9.1\%) \\
\hline
\end{tabular}

In a one-arm study conducted by Lan CY et al in $2018,{ }^{24}$ apatinib combined with etoposide was used to treat platinum-resistant ovarian cancer patients, and the tumors in 29 out of 31 patients (94\%) decreased, 19 patients achieved PR (61\%), DCR was 97\%.

This study eventually included 22 patients with advanced ovarian cancer with an overall RR of $20 \%$ and a DCR of $85 \%$. A total of 6 patients $(50 \%)$ in the combined group had a reduction in tumors, and a total of 3 patients $(35 \%)$ in the monotherapy group had a decrease in tumors. The effectiveness of the Lan CY study was more significant than this study. ${ }^{24}$ The reason was considered that the sample size of the study was small, as well as to be related to the retrospective study and the inconsistency of the combination of chemotherapy drugs.

The study also showed that RR and DCR were significantly higher in the combined group than the monotherapy group, PFS and OS were higher than the monotherapy group, but the $\mathrm{P}$ value was greater than 0.05 . Considering that the overall sample size is small, and this study is a retrospective study, more cases are needed for prospective studies. Only one patient in this study had previously received bevacizumab for economic reasons, apatinib combined with topotecan was applied after the disease progression, followed by PR again. This suggests that apatinib treatment may still be considered when there is a disease progression following anti-angiogenic therapy with bevacizumab.

The currently marketed PARP inhibitors include olaparib, rucaparib and niraparib, which are mainly used for platinum-sensitive ovarian cancer patients and are expensive. The patients in this study were all platinum-resistant patients with advanced ovarian cancer, while apatinib was cheaper and had a better pharmacoeconomic effect.

Some evidence suggests that the management of ovarian cancer should be personalized taking into account the performance status of the patient, in particular in case of elderly women. ${ }^{25-27}$ In this study, the average age of the patients was 55.6 years, and the maximum age was 79 years. The majority of patients showed good tolerance to apatinib. The incidence of proteinuria and hand and foot skin reactions in the combined group was significantly higher than in the apatinib monotherapy group. We need to pay close attention to the adverse reactions of apatinib. A meta-analysis showed that the incidence of adverse reactions to apatinib was $84 \%$ in cancer treatment ${ }^{28}$ and $100 \%$ in ovarian cancer treatment, ${ }^{24}$ but most of them were 1-2 grades of toxicity. The incidence of adverse reactions in this study was $100 \%$, most of them were 1-2 grade adverse reactions, and fewer 3-4 grades adverse reactions, mainly hypertension and hand and foot skin reactions, which was consistent with previous studies. Hypertensive patients can be administered with ACEI or ARB drugs to lower blood pressure. ${ }^{22}$ Topical hydrocortisone ointment or urea ointment can be used for the treatment of hand and foot skin reactions, the combination of oral vitamin $\mathrm{B}$ treatment is more effective. Therefore, the adverse reactions should be strictly observed in the course of treatment, and symptomatic treatment should be applied and preventive measures should be strengthened. In general, 
there were many adverse reactions of apatinib, but most of these adverse reactions are reversible and controllable.

Apatinib is a new option after failure of multi-line treatment for ovarian cancer, but it is still in the exploratory stage, and currently apatinib is only used to treat Asian population with ovarian cancer, and its efficacy in other communities has not yet been defined. Therefore, further research is needed to provide more data and ultimately apply it to clinical practice.

\section{Author Contributions}

All authors contributed to data analysis, drafting or revising the article, gave final approval of the version to be published and agree to be accountable for all aspects of the work.

\section{Funding}

This work was supported by the National Natural Science Foundation of China (No.81272741), the Project of Making Health Become Strong by Science and Teaching at the Health and Family Planning Commission in Jiangsu Province (QNRC2016042), Nanjing Medical Science and Technique Development Foundation (Outstanding Youth Foundation, JQX14001) and Medical Scientific Research Project of Jiangsu Provincial Health and Family Planning Commission (z2018046).

\section{Disclosure}

The authors report no conflicts of interest in this work.

\section{References}

1. Bray F, Ferlay J, Soerjomataram I, et al. Global cancer statistics 2018: GLOBOCAN estimates of incidence and mortality worldwide for 36 cancers in 185 countries. CA Cancer J Clin. 2018. doi:10.3322/ caac. 21492

2. Grossman DC, Curry SJ, Owens DK, et al. Screening for ovarian cancer: US preventive services task force recommendation statement JAMA. 2018;319(6):588-594. doi:10.1001/jama.2017.21926

3. Chen W, Zheng R, Baade PD, et al. Cancer statistics in China, 2015. CA Cancer J Clin. 2016;66(2):115-132. doi:10.3322/caac.21338

4. Korkmaz T, Seber S, Basaran G. Review of the current role of targeted therapies as maintenance therapies in first and second line treatment of epithelial ovarian cancer; in the light of completed trials. Crit Rev Oncol Hematol. 2016;98:180-188. doi:10.1016/j.critrevonc.2015. 10.006

5. Li J, Qin S, Xu J, et al. Randomized, double-blind, placebo-controlled Phase III trial of apatinib in patients with chemotherapy-refractory advanced or metastatic adenocarcinoma of the stomach or gastroesophageal junction. J Clin Oncol. 2016;34(13):1448-1454. doi:10.1200/ JCO.2015.63.5995

6. Gong XL, Qin SK. Progress in systemic therapy of advanced hepatocellular carcinoma. World J Gastroenterol. 2016;22(29):6582-6594. doi:10.3748/wjg.v22.i29.6582
7. Qin SK. Apatinib in Chinese patients with advanced hepatocellular carcinoma: a phase II randomized, open-label trial. J Clin Oncol. 2014;32 Suppl 5:abstract4019. doi:10.1200/jco.2014.32.15_suppl.4019

8. Pujade-Lauraine E, Hilpert F, Weber B, et al. Bevacizumab combined with chemotherapy for platinum-resistant recurrent ovarian cancer: the AURELIA open-label randomized phase III trial. J Clin Oncol. 2014;32:1302-1308. doi:10.1200/JCO.2013.51.4489

9. Du Bois A, Floquet A, Kim JW, et al. Incorporation of pazopanib in maintenance therapy of ovarian cancer. J Clin Oncol. 2014;32:33743382. doi:10.1200/JCO.2014.55.7348

10. Laganà AS, Sofo V, Vitale SG, Triolo O. Epithelial ovarian cancer inherent resistance: may the pleiotropic interaction between reduced immunosurveillance and drug-resistant cells play a key role. Gynecol Oncol Rep. 2016;18:57-58. doi:10.1016/j.gore.2016.09.004

11. Laganà AS, Colonese F, Colonese $\mathrm{E}$, et al. Cytogenetic analysis of epithelial ovarian cancer's stem cells: an overview on new diagnostic and therapeutic perspectives. Eur J Gynaecol Oncol. 2015;36(5):495-505.

12. Hamerlik P, Lathia JD, Rasmussen R, et al. Autocrine VEGFVEGFR2-Neuropilin-1 signaling promotes glioma stem-like cell viability and tumor growth. J Exp Med. 2012;209(3):507-520. doi:10.1084/jem.20111424

13. Burger RA, Brady MF, Rhee J, et al. Independent radiologic review of the Gynecologic Oncology Group Study 0218, a phase III trial of bevacizumab in the primary treatment of advanced epithelial ovarian, primary peritoneal, or fallopian tube cancer. Gynecol Oncol. 2013;131(1):21-26. doi:10.1016/j.ygyno.2013.07.100

14. Burger RA, Brady MF, Bookman MA, et al. Incorporation of bevacizumab in the primary treatment of ovarian cancer. $N$ Engl $J$ Med. 2011;365(26):2473-2483. doi:10.1056/NEJMoa1104390

15. Coleman RL, Brady MF, Herzog TJ, et al. Bevacizumab and paclitaxel-carboplatin chemotherapy and secondary cytoreduction in recurrent, platinum-sensitive ovarian cancer (NRG Oncology/ Gynecologic Oncology Group study GOG-0213): a multicentre, open-label, randomised, phase 3 trial. Lancet Oncol. 2017;18 (6):779-791. doi:10.1016/S1470-2045(17)30279-6

16. Pujade-Lauraine E, Ledermann JA, Selle F, et al. Olaparib tablets as maintenance therapy in patients with platinum-sensitive, relapsed ovarian cancer and a BRCA1/2 mutation (SOLO2/ ENGOT-Ov21): a double-blind, randomised, placebo-controlled, phase 3 trial. Lancet Oncol. 2017;18(9):1274-1284. doi:10.1016/ S1470-2045(17)30469-2

17. Coleman RL, Oza AM, Lorusso D, et al. Rucaparib maintenance treatment for recurrent ovarian carcinoma after response to platinum therapy (ARIEL3): a randomised, double-blind, placebo-controlled, phase 3 trial. Lancet. 2017;390(10106):1949-1961. doi:10.1016/ S0140-6736(17)32440-6

18. Moore KN, Vergote I, Oaknin A, et al. FORWARD I: a Phase III study of mirvetuximabsoravtansine versus chemotherapy in platinumresistant ovarian cancer. Future Oncol. 2018;14(17):1669-1678. doi:10.2217/fon-2017-0646

19. Moore KN, O'Malley DM, Vergote I, et al. Safety and activity findings from a phase $1 \mathrm{~b}$ escalation study of mirvetuximabsoravtansine, a folate receptor alpha ( $F R \alpha$ )-targeting antibody-drug conjugate (ADC), in combination with carboplatin in patients with platinumsensitive ovarian cancer. Gynecol Oncol. 2018;151(1):46-52. doi:10.1016/j.ygyno.2018.07.017

20. Moore KN, Martin LP, O'Malley DM, et al. Safety and activity of mirvetuximabsoravtansine (IMGN853), a folate receptor alphatargeting antibody-drug conjugate, in platinum-resistant ovarian, fallopian tube, or primary peritoneal cancer: a Phase I expansion study. J Clin Oncol. 2017;35(10):1112-1118. doi:10.1200/JCO. 2016.69.9538

21. Zhang H. Apatinib for molecular targeted therapy in tumor. Drug Des Devel Ther. 2015;9:6075-6081.

22. Qin SK, Li J. Clinical expert consensus of gastric carcinoma treated with apatinib. Chin Clinl Oncol. 2015;9:841-847. 
23. National Comprehensive Cancer Network, Inc. NCCN clinical practice guidelines in oncology: gastric cancer (Version2.2015). 2015. Available from: http://www.nccn.org/professionals/physician_gls/ pdf/gastric.pdf. Accessed September 26, 2019.

24. Lan CY, Wang Y, Xiong Y, et al. Apatinib combined with oral etoposide in patients with platinum-resistant or platinum-refractory ovarian cancer (AEROC): a phase 2, single-arm, prospective study. Lancet Oncol. 2018;19(9):1239-1246. doi:10.1016/S1470-2045(18)30349-8

25. Vitale SG, Capriglione S, Zito G, et al. Management of endometrial, ovarian and cervical cancer in the elderly: current approach to a challenging condition. Arch Gynecol Obstet. 2019;299(2):299-315. doi:10.1007/s00404-018-5006-z
26. Schuurman MS, Kruitwagen R, Portielje J, et al. Treatment and outcome of elderly patients with advanced stage ovarian cancer: a nationwide analysis. Gynecol Oncol. 2018;149(2):270-274. doi:10. 1016/j.ygyno.2018.02.017

27. Bellia A, Vitale SG, Laganà AS, et al. Feasibility and surgical outcomes of conventional and robot-assisted laparoscopy for early-stage ovarian cancer: a retrospective, multicenter analysis. Arch Gynecol Obstet. 2016;294(3):615-622. doi:10.1007/s00404-016-4087-9

28. Sun D, Hou H, Zhang C, Zhang X. The efficacy and safety of apatinib for refractory malignancies: a review and metaanalysis. Onco Targets Ther. 2018;11:6539-6554. doi:10.2147/ OTT.S 176429

\section{Publish your work in this journal}

Cancer Management and Research is an international, peer-reviewed open access journal focusing on cancer research and the optimal use of preventative and integrated treatment interventions to achieve improved outcomes, enhanced survival and quality of life for the cancer patient.
The manuscript management system is completely online and includes a very quick and fair peer-review system, which is all easy to use. Visit http://www.dovepress.com/testimonials.php to read real quotes from published authors. 